\title{
DAÑo OCASIONADO POR EL FALSO GUSANO ALAMBRE Eleodes pos. omissoides Blaisdell (COLEOPTERA: TENEBRIONIDAE) EN SEMILLAS DE GRAMÍNEAS Y LEGUMINOSAS
}

\section{DAMAGE OF THE FALSE WIREWORM Eleodes pos. omissoides Blaisde/l (Coleoptera: Tenebrionidae) UPON SEEDS OF GRASSES AND LEGUMES}

\author{
Daniel Quiroga-Murcia ${ }^{1}$, Francisco Javier Posada-Flórez ${ }^{2}$ \\ ${ }^{1}$ Joven investigador, Estudiante de Ingeniería Agronómica, Universidad de Ciencias Aplicadas y Ambientales, U.D.C.A, Calle \\ 222 No. 55-37, Bogotá, D.C., Colombia, quirogaedaniel@hotmail.com; ${ }^{2}$ Ing. Agrónomo, Ph.D. Profesor Entomólogo. Uni- \\ versidad de Ciencias Aplicadas y Ambientales U.D.C.A hasta Junio de 2012, fjavierposada@hotmail.com
}

Rev. U.D.C.A Act. \& Div. Cient. 16(2): 391-400, Julio-Diciembre, 2013

\section{RESUMEN}

Tanto los adultos como las larvas del falso gusano alambre (Eleodes pos. omissoides) viven en el suelo y se alimentan de semillas, causando faltantes en los cultivos. Esto afecta los rendimientos e incrementa los costos de producción, debido a las medidas de manejo. Se evaluó el daño que causan las larvas sobre las semillas de arveja, de maíz y de trigo, secas e hidratadas, haciendo observaciones cada seis horas hasta las setenta y dos, para determinar el tipo de lesión. En cada observación, se registró si la semilla permanecía intacta, si era atacado su endospermo, si su embrión era destruido completamente. Los resultados indicaron que todas las semillas sufrieron lesiones; inmediatamente, se colocaron en el suelo. La afectación fue variable dependiendo de si la semilla estaba seca o hidratada. Las semillas de la arveja presentaron menor daño, seguidas por las del maíz y las del trigo, tanto secas como hidratadas. Por tipo de lesión, el ataque al embrión presentó el mayor valor, seguido por el causado al endospermo y, en menor cantidad, la destrucción total de la semilla. En general, el daño de las semillas alcanzó un promedio de 50,64\%. Aunque germinaron las semillas cuyo endospermo estaba afectado, se presentó la colonización de este por hongos.

Palabras clave: Suelo, siembra, pérdida, germinación, hongos.

\section{SUMMARY}

Adults and larvae of the false wireworm Eleodes pos omissoides live in the soil and feed on seeds. This habit cause losses on the crops, decreases yield and increases the production cost as control actions needs to be applied. The damage caused by the larvae on pea, corn and wheat seeds were evaluated, offering dry and during 24 hours water hydrated seeds before exposition to the larvae. Evaluation to record the damage type was done every six hours for a period of 72 hours. During the evaluations it was established if the seed remained free of damage, if the endosperm was attacked, if the embryo or the whole seed was consumed. The results showed that all seeds were attacked as soon as they were exposed to the larvae in the soil. The response to the damage was variable, depending on the exposure of dry or hydrated seeds. The pea seeds presented the lowest damage, followed by corn, being the most susceptible ones wheat, both dry and hydrated. Regarding the type of damage the attacked embryo showed the highest value, followed by the damage to the endosperm and in lesser amount the destruction of the whole seed. Overall, the damage of the seeds reached on average $50.64 \%$. Seeds that had suffered endosperm damage germinated, but showed fungal development.

Key words: Soil, seeding, losses, germination, fungi.

\section{INTRODUCCIÓN}

El falso gusano alambre (Eleodes pos. omissoides Blaisdell (Coleoptera: Tenebrionidae) es una especie plaga registrada en el país, en Cundinamarca y en Boyacá, como lo demuestran las referencias adjuntas en Notas y Noticias Entomológicas (Vergara \& Varela, 1977; Posada et al. 1980; Zenner de 
Polanía \& Borrero, 1992; López et al. 1995). La zona donde se ha observado este insecto pertenece a la formación vegetal de bosque seco tropical montano bajo (bs-MB) (Espinal \& Montenegro, 1977), cuyas condiciones ecológicas favorecen, aparentemente, a las poblaciones de esta especie.

La mayor diversidad de insectos de la familia Tenebrionidae, se presenta en zonas secas y en suelos de textura arenosa en el medio oeste de los Estados Unidos, donde estos escarabajos son conocidos como los coleópteros del desierto (Doyen \& Tschinkel, 1974; Mclntyre, 1998). En Colombia, la fauna de Tenebrionidae es escasamente estudiada y se desconoce la mayor parte de los aspectos de biología, de ecología e importancia económica en la agricultura.

Los registros de $E$. pos. omissoides en el país indican que ataca las semillas en la época de siembra y el daño es causado, tanto por la larva como por el adulto. Cuando se presenta en invernaderos causa pérdidas de semillas de arveja y de maíz, que alcanzan el 80 \% (Quiroga \& Posada, datos aún no publicados). En campo, se han registrado pérdidas en cultivos de maíz del $20 \%$; además, los adultos se han observado atacando hortalizas, como el tomate, la cebolla, la espinaca y la papa (Posada et al. 1980; Zenner de Polanía \& Borrero, 1992; Zenner de Polanía, 1994b; López et al. 1995; Zenner de Polanía et al. 1999); también consumen follaje de papa y de arveja en el estado de plántulas (Posada et al. 1990b; Zenner de Polanía \& Borrero, 1992; Zenner de Polanía, 1994a).

Los hábitos alimenticios de las larvas y de los escarabajos son muy variados. Adicional a que son fitófagos, se ha notado que sobreviven sin alimento, consumiendo materia orgánica del suelo, como detritívoros (Posada et al. 1987; 1988;
McIntyre, 1998). Además, se ha observado que son depredadores de otros insectos y moluscos presentes en el suelo y muestran comportamientos canibalísticos, en los cuales, las larvas se atacan entre sí y los adultos consumen larvas y huevos de la misma especie (Quiroga \& Posada, datos aún no publicados).

Los adultos pueden invadir viviendas y se ocultan en sitios oscuros, donde la presencia y el olor que secretan causan malestar a los habitantes. Igualmente, en cultivos de consumo fresco, como la lechuga en época de cosecha, afectan la comercialización en campo, cuando los compradores observan las altas poblaciones de este insecto en el cultivo (Posada et al. 1988).

Los falsos gusano alambre son considerados como tierreros, que se alimentan de las semillas y de las raíces tiernas de las plantas; no obstante, en la etapa adulta, se apartan de este comportamiento, ya que actúan más como ladrones de semilla, porque las remueven del sitio de siembra y las desplazan a otro lugar, donde las utilizan como alimento. Igualmente, las larvas, cuando encuentran las semillas, las profundizan en el suelo hasta que las consumen totalmente (Quiroga \& Posada, datos aún no publicados) y crean los faltantes en el cultivo, que obligan a la resiembra, lo que genera incremento de costos, por el valor de la semillas y la mano de obra que se requiere para la labor.

Los adultos (Figura 1) son de tamaño grande $(2,21 \pm$ $0,18 \mathrm{~cm})$, de color negro, de hábitos gregarios y no vuelan; son muy activos en la noche y en el día permanecen refugiados bajo residuos vegetales o escombros (Quiroga \& Posada, datos aún no publicados). Comúnmente, los adultos se conocen como escarabajos olorosos o bombarderos,

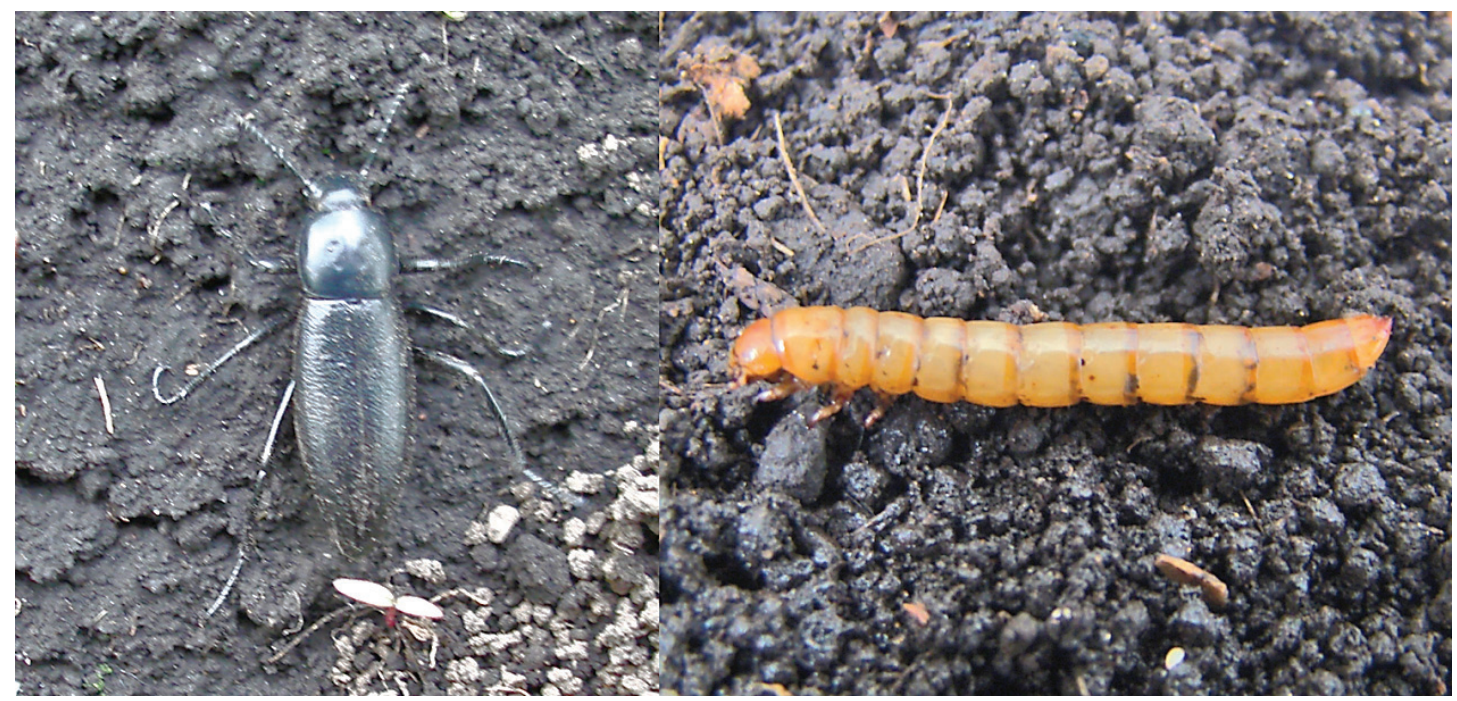

Figura 1. Larva y adulto del falso gusano alambre Eleodes pos. omissoides, en su hábitat natural, el suelo. 
porque cuando se disturban, secretan una sustancia de olor nauseabundo, probablemente, para protegerse de los depredadores (Mclntyre, 1998).

Las larvas (Figura 1) habitan dentro del suelo; se sabe que son fotofóbicas, porque cuando se exponen a la luz buscan enterrase rápidamente. La denominación de falso gusano alambre, se debe a que las larvas son muy similares a las del gusano alambre, que pertenece a los coleópteros de la familia Elateridae; se presentan en el suelo atacando gran diversidad de cultivos, actuando como tierreros y tienen amplia distribución geográfica (Metcalf \& Flint, 1962; Posada, 1989; Toba, 1985).

El insecto ha mostrado preferencia por atacar semillas de maíz, de arveja y de trigo. El suministro de estas semillas a adultos en el laboratorio colocados en recipientes con tierra permitió la cría masiva de este insecto. Los adultos son insectos muy longevos, en cautiverio se han logrado mantener vivos por dos años en cajas con suelo húmedo y alimentados con semillas de trigo (Posada et al. 1990a; Quiroga \& Posada, datos aún no publicados).

El conocimiento apropiado del daño causado por los insectos y su evaluación es uno de los pilares del manejo integrado de plagas. Debido a la escasa información sobre el falso gusano alambre, como insecto plaga, en este trabajo se tuvo como objetivo, evaluar el daño ocasionado por las larvas del gusano alambre sobre semillas de arveja, maíz y trigo, secas e hidratadas, colocadas en el suelo. Además, se evaluó la sobrevivencia de las semillas luego de la afectación.

\section{MATERIALES Y MÉTODOS}

El trabajo, se realizó en el laboratorio de entomología de la Universidad de Ciencias Aplicadas y Ambientales U.D.C.A, localizada en el norte de la sabana de Bogotá, con coordenadas N 447'57'- W 7402'40', temperatura media anual de $14,5^{\circ} \mathrm{C}$, precipitación media anual de $860 \mathrm{~mm}$ con régimen bimodal y altura de $2570 \mathrm{msnm}$.

Semillas: Se tomaron cuarenta semillas secas e hidratadas (durante 24 horas) de arveja (Pisum sativum L., Fabaceae), variedad Santa Isabel; de maíz (Zea mays L., Poaceae), hibrido ICA355 y de trigo (Triticum aestivum L., Poaceae), ICA Yacuanqer y se expusieron a larvas del insecto, para estimar el daño. Se realizó un lavado previo de todas las semillas, para eliminar residuos de químicos e insecticidas que pudiesen afectar a las larvas.

Larvas del gusano alambre: Se colectaron adultos del insecto en la sede El Remanso de la U.D.C.A, donde se presentan abundantemente como plaga de cultivos, en especial, de maíz y de arveja. Los individuos colectados, se trasladaron al laboratorio de entomología y se colocaron en cajas plásticas (Viniplast 25x30x12cm), en las cuales, se les adicionó un kilo de suelo orgánico, que se mantuvo húmedo, para recrear las condiciones ambientales, donde ellos se mantuvieron, facilitando la alimentación y la reproducción. La cría y la evaluación de las semillas, se desarrollaron en condiciones del laboratorio, con temperatura promedio de $15^{\circ} \mathrm{C}$. De las posturas colocadas por estos coleópteros en el laboratorio, se obtuvieron las larvas, que se conservaron en suelo orgánico y se les adicionó semillas de trigo, como alimento, hasta que alcanzaron un tamaño medio de dos centímetros, para ser utilizadas en el experimento.

Evaluación del daño. Las semillas, se revisaron cada seis horas, para establecer el tipo de lesión y determinar el número de semillas dañadas y el tiempo en que eran afectadas por la alimentación de las larvas y si los remanentes de las semillas mostraban capacidad de germinación o de crecimiento de hongos, por efecto del daño. Se evaluó como cero, si la semilla permanecía en perfecto estado; uno, si el embrión presentaba lesión; dos, si el endospermo era dañado y tres, si la semilla era consumida totalmente (Figura 2). El tiempo de evaluación de las semillas se extendió por 72 horas, lapso en el que se realizaron, cada seis horas, 13 evaluaciones, incluyendo el tiempo cero o inicio del experimento, en el que todas las semillas estaban ilesas. En total, por cada tratamiento y tiempo de evaluación, se realizaron 260 observaciones y para todo el experimento, un total de 1.560 .

Experimento: Se evaluaron seis tratamientos con tres tipos de semillas (arveja, maíz y trigo) expuestas secas o hidratadas (por 24 horas). Se organizó en un diseño completamente al azar, con 10 repeticiones, cada una conformada por dos larvas y dos semillas, colocadas en recipientes de plástico (AcmeLeon D30482), con $6 \mathrm{~g}$ de suelo húmedo, que cubrió las semillas y las larvas. En total, por cada tratamiento, se evaluaron 20 semillas y 20 larvas.

Los resultados, se analizaron estadísticamente, utilizando SAS versión 9.3 (SAS Institute Inc. 2009). En el análisis de varianza, se utilizó el Proc Glimix, que normaliza los datos y cuando se encontraron diferencia significativas, se evaluaron los contrastes, estimando la media de los mínimos cuadrados (Lsmeans = least square means) de los tratamientos. La diferencia entre los tratamientos, se consideró significante, cuando $P<0.05$. Adicionalmente, se calculó la frecuencia y construyeron tablas de contingencia, utilizando Chi cuadrada por semilla, estado (seca e hidratada) y del tipo lesión. Los tiempos de evaluación por semilla y estado se graficaron, para establecer la intensidad del daño y conocer el tiempo crítico, para tomar decisiones de resiembra. 


\begin{tabular}{|c|c|c|c|c|c|}
\hline Semillla & $\begin{array}{l}\text { Sin } \\
\text { daño }\end{array}$ & $\begin{array}{l}\text { Daño } \\
\text { Endos- } \\
\text { permo }\end{array}$ & $\begin{array}{l}\text { Daño } \\
\text { Embrión }\end{array}$ & $\begin{array}{l}\text { Con Daño } \\
\text { Completo }\end{array}$ & $\begin{array}{l}\text { Daño } \\
\text { secun- } \\
\text { dario }\end{array}$ \\
\hline & 0 & 1 & 2 & 3 & \\
\hline Arveja & & & & & \\
\hline Maíz & & & & & \\
\hline Trigo & & & & & \\
\hline
\end{tabular}

Figura 2. Escala para la evaluación del daño de las larvas del falso gusano alambre Eleodes pos. Omissoides, sobre semillas de arveja, de maíz y de trigo.

\section{RESULTADOS Y DISCUSIÓN}

Todas las semillas de arveja, de maíz y de trigo expuestas a las larvas del falso gusano alambre sufrieron daño (Figura 3, Tablas 1 y 2) y, en general, en el análisis de varianza por semilla, se encontró diferencias significativas $(\mathrm{GL}=2,1548$, $\mathrm{F}=9.11, \mathrm{P}=0.0001)$. El trigo presentó la mayor cantidad de semillas afectadas (promedio \pm error estándar), 63,1 \pm 1,97, seguido por el maíz. 51,3 \pm 1,97 y la arveja, 37,5 \pm 1,97. Igualmente, en general, la evaluación del daño de las semillas por estado, secas e hidratadas, presentó diferencias significativas ( $\mathrm{GL}=1,1548, \mathrm{~F}=4,14, \mathrm{P}=0,0421$ ), en las semillas secas fue mayor, que llegó a 53,3 $\pm 1,61$, mientras que en las hidratadas alcanzó el 47,9 \pm 1,61 \% (Tabla 2).

En la interacción del daño sobre las semillas y el estado (secas o hidratadas), se presentaron diferencias significativas $(\mathrm{Gl}=2,1548, \mathrm{~F}=6,01, \mathrm{P}=0,0025)$ y se evidenció gran variación, ya que fluctuó entre $25 \pm 2,78$, para la arveja seca y 70,4 $\pm 2,78$, para el trigo seco (Tabla 2). De estos resultados, se puede inferir que las larvas del falso gusano alambre presentaron preferencia por semillas de trigo y de maíz $\mathrm{y}$, adicionalmente, el estado de estas, también influye en el comportamiento del daño, puesto que las semillas secas mostraron mayores ataques.

El análisis por cada tiempo en que se realizaron las observaciones presentó una respuesta diferente por la semilla y el estado (seca e hidratada). Tan pronto como las semillas se colocaron y se cubrieron con el suelo, los insectos comenzaron a alimentarse de éstas (Figura 3). El daño sobre la arveja frente al maíz y el trigo estuvo influenciada por el estado de la semilla; sin embargo, en el análisis de varianza no se presentó diferencia significativa para las interacciones de la semilla por estado y por cada tiempo de evaluación $(\mathrm{GL}=2$, $1548, F=2,34, P=0,0965$ ) (Tabla 3). El tiempo o rapidez con que se presentó el daño por semilla y estado (Figura 3) mostró que la arveja seca sufrió, inicialmente, menos que la hidratada, aunque al final (72 horas), se presentó igual cantidad de daño (60\%). Lo contrario ocurrió para las semillas de maíz y de trigo, donde las secas sufrieron mayor y más rápido el daño, lo que indica que las Gramíneas o Poaceas son más susceptibles que las leguminosas o Fabaceas bajo las condiciones experimentales evaluadas.

Comparando el daño al tiempo inicial y final, se encontró que a las seis horas las semillas de arveja, seca e hidratada, presentaron 10 y $40 \%$ de daño y a las 72 horas alcanzó el $60 \%$, para ambas. En las semillas de maíz, secas e hidratadas, a las seis horas, el 25 y 35\%, respectivamente, estaban afectadas y, a las 72 horas, presentó niveles de 90 y $45 \%$. En las semillas de trigo, el daño a las seis horas, alcanzó 50 y $55 \%$, respectivamente y, a las 72 horas, reveló niveles de 90 y $65 \%$. En general, el ataque del falso gusano alambre presentó la tendencia a ser más severa sobre las semillas de maíz y de trigo secas, al contrario de la arveja, donde las semillas sufrieron el daño más rápido y severo (pérdida de las semillas) (Figura 2, Tabla 2). También, se encontró que las 

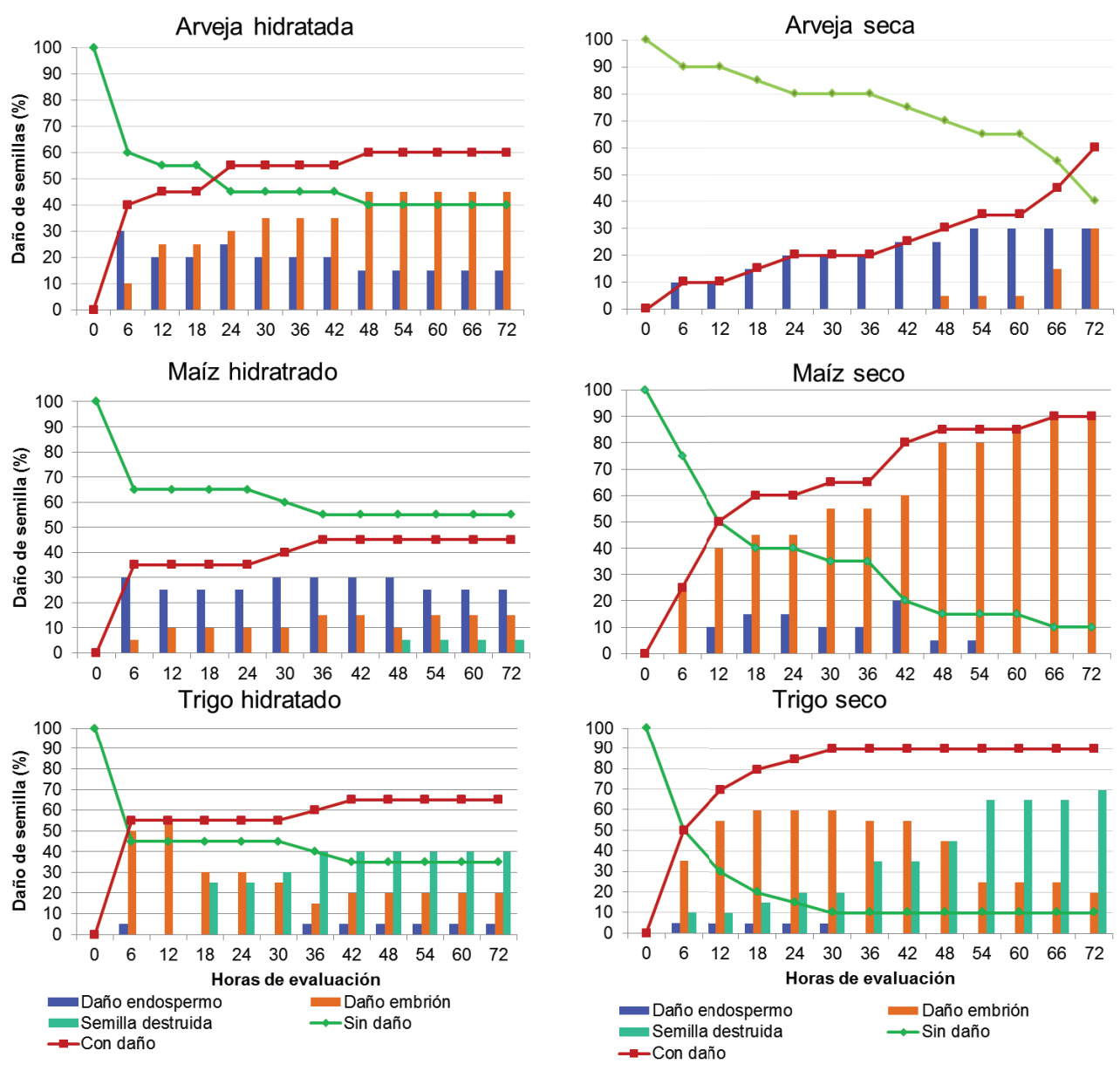

Figura 3. Evaluación del tiempo de daño causado por larvas del falso gusano alambre, sobre semillas de arveja, de maíz y de trigo, expuestas secas e hidratadas.

semillas secas mostraron la tendencia a sufrir, inicialmente, menos que las hidratadas, pero al final de la evaluación las de maíz y de trigo secas fueron las más afectadas. El comportamiento de la respuesta al daño dependiendo del estado de la semilla es un aspecto que merece mayor investigación, para establecer si esta puede ser influenciada por la variedad de la semilla y las condiciones de materia orgánica y humedad del suelo.

En maíz y en trigo, la hidratación de las semillas tuvo un efecto positivo, reduciendo la cantidad de semillas dañadas y desaparecidas, por el ataque de las larvas. La evaluación demostró que en estas semillas, la hidratación puede disminuir la incidencia en 45 y 30\%, respectivamente, en relación con las secas, lo cual, en la práctica, puede ser una recomendación técnica para el manejo del falso gusano alambre, en cultivos de gramíneas, cuando el suelo de los lotes presenta, históricamente, larvas del falso gusano alambre (Figura 3, Tabla 1,3).
En la evaluación del daño sobre las partes de las semillas atacadas, considerando el estado, secas e hidratadas, se encontró que en las semillas de arveja (seca e hidratada) y en las de maíz (secas), el daño solamente se causó sobre el endospermo y el embrión, mientras que en las de maíz (hidratado) y en las de trigo (seco e hidratado), se presentó pérdida de endospermos, del embrión y la destrucción total de las semillas (Figura 3, Tabla 1).

En la arveja seca, la lesión sobre el endospermo reveló una tendencia creciente y alcanzó el mayor valor (60\%), a las 72 horas. Sobre el embrión, solamente se inició el daño al cabo de las 48 horas (5\%) y, a las 72 horas, llegó al (30\%). En las semillas de arveja hidratadas, el daño sobre el endospermo y el embrión, se presentó a partir de las seis horas, donde en el endospermo fue mayor (30\%) y decreció hasta las 72 horas (15\%). Lo contrario ocurrió con el daño del embrión, que mostró una tendencia a aumentar hasta las 72 horas (45\%). 
Tabla 1. Evaluación de la frecuencia del daño de las semillas de arveja, de maíz y de trigo expuestas secas e hidratadas, a larvas del falso gusano alambre por 72horas.

\begin{tabular}{|c|c|c|c|c|c|}
\hline \multirow{2}{*}{$\begin{array}{l}\text { Semilla x estado } \\
\text { Frecuencia } \\
\text { Porcentaje } \\
\text { Porcentaje linea } \\
\text { Porcentaje columna }\end{array}$} & \multicolumn{5}{|c|}{ Daño } \\
\hline & $\begin{array}{c}\text { Sin daño } \\
0\end{array}$ & $\begin{array}{c}\text { Daño } \\
\text { endospermo } \\
1\end{array}$ & $\begin{array}{c}\text { Daño embrión } \\
2 \\
\end{array}$ & $\begin{array}{c}\text { Con Daño } \\
\text { completo } \\
3 \\
\end{array}$ & Total \\
\hline Anveja Hidratada & $\begin{array}{c}130 \\
8,33 \\
50,00 \\
18,89 \\
\end{array}$ & $\begin{array}{c}46 \\
2,95 \\
17,69 \\
23,71 \\
\end{array}$ & $\begin{array}{c}84 \\
5,38 \\
32,31 \\
19,44 \\
\end{array}$ & $\begin{array}{c}0 \\
0.00 \\
0.00 \\
0.00\end{array}$ & $\begin{array}{c}260 \\
16,67\end{array}$ \\
\hline Arveja Seca & $\begin{array}{c}195 \\
12,50 \\
75,00 \\
25,32 \\
\end{array}$ & $\begin{array}{c}53 \\
3,40 \\
20,38 \\
27,32 \\
\end{array}$ & \begin{tabular}{c|}
12 \\
0,77 \\
4,62 \\
2,78 \\
\end{tabular} & $\begin{array}{c}0 \\
0,00 \\
0,00 \\
0,00 \\
\end{array}$ & $\begin{array}{c}260 \\
16,67\end{array}$ \\
\hline Maiz Hidratado & $\begin{array}{c}161 \\
10,32 \\
61,92 \\
20,91 \\
\end{array}$ & $\begin{array}{c}65 \\
4,17 \\
25,00 \\
33,51 \\
\end{array}$ & $\begin{array}{c}29 \\
1,86 \\
11,15 \\
6,71 \\
\end{array}$ & $\begin{array}{c}5 \\
0,32 \\
1,92 \\
3,05 \\
\end{array}$ & $\begin{array}{c}260 \\
16,67\end{array}$ \\
\hline Maiz Seco & $\begin{array}{c}92 \\
5,9 \\
35,38 \\
11,95 \\
\end{array}$ & $\begin{array}{c}18 \\
1,15 \\
6,92 \\
9,28 \\
\end{array}$ & $\begin{array}{c}150 \\
9,62 \\
57,69 \\
34,72 \\
\end{array}$ & $\begin{array}{c}0 \\
0,00 \\
0,00 \\
0,00 \\
\end{array}$ & $\begin{array}{c}260 \\
16,67\end{array}$ \\
\hline Trigo Hidratado & $\begin{array}{c}115 \\
7,37 \\
44,23 \\
14,94 \\
\end{array}$ & $\begin{array}{c}8 \\
0,51 \\
3,08 \\
4,12 \\
\end{array}$ & \begin{tabular}{c|}
65 \\
4,17 \\
25,00 \\
15,05 \\
\end{tabular} & $\begin{array}{c}72 \\
4,62 \\
27,69 \\
43,90 \\
\end{array}$ & $\begin{array}{c}260 \\
16,67\end{array}$ \\
\hline Trigo seco & $\begin{array}{c}77 \\
4,94 \\
29,62 \\
10,00 \\
\end{array}$ & $\begin{array}{c}4 \\
0,26 \\
1,54 \\
206 \\
\end{array}$ & $\begin{array}{c}92 \\
5,90 \\
35,38 \\
21,30 \\
\end{array}$ & $\begin{array}{c}87 \\
5,58 \\
33,46 \\
53,01 \\
\end{array}$ & $\begin{array}{c}260 \\
16,67\end{array}$ \\
\hline Total & $\frac{770}{49,36}$ & $\frac{194}{12,44}$ & $\frac{432}{27.69}$ & $\frac{164}{10.51}$ & $\frac{1560}{100.00}$ \\
\hline
\end{tabular}

Freq. Proc. $\chi^{2}=648,56 ;$ G.L. $\left.=15 ; P=0,0001\right)$.

En maíz, con las semillas secas, las larvas mostraron mayor preferencia por atacar el embrión que el endospermo, contrario a las semillas hidratadas. En las semillas secas, el daño al embrión empezó a las seis horas (25\%) y se incrementó hasta las 72 horas (90\%), mientras que sobre el endospermo solamente se empezó a presentar a las 12 horas (10\%) y se extendió hasta las 54 horas (5\%). En las semillas hidratadas, el daño del endospermo y del embrión, se evidenció a partir de las seis horas y se mantuvo casi constante hasta las 72 horas, con valores de $25 \%$, para el endospermo y $15 \%$, para el embrión. En las semillas hidratadas, la pérdida total de semillas se detectó a partir de las 48 horas.

En trigo, la mayor incidencia se observó sobre el endospermo, el embrión y, además, ocurrió la pérdida total de la semilla, lo que puede indicar mayor preferencia de las larvas del insecto por las semillas de trigo, frente a las demás evaluadas. En las semillas de trigo secas, se detectaron todos los tipos de lesión, a partir de las seis horas. El daño sobre el endospermo fue el más bajo (5\%) y se mantuvo constante hasta las 24 horas; sobre el embrión, inicialmente, alcanzó un nivel de $35 \%$, se incrementó rápidamente hasta el $60 \%$, a las 24 horas y decreció hasta $20 \%$, a las 72 horas, esto se explica porque cambiaba la catalogación de la lesión y pasaba a grado 3 (pérdida total). La pérdida total de la semilla, a las seis horas, presentó un $10 \%$ y se incrementó hasta un $70 \%$, a las 72 horas. Igualmente, las semillas hidratadas sufrieron todos los tipos de lesión, pero exhibieron diferente respuesta. A las seis horas, únicamente se había presentado daño en el endospermo (5\%), que se mantuvo hasta las 72 horas y en el embrión (50\%). El mayor porcentaje de daño en el embrión (55\%), se presentó a las 12 horas y decreció a las 72 horas, 
Tabla 2. LSmeans* de la respuesta general del daño causado por larvas del falso gusano alambre, por semillas, por estado, seca e hidratada y por la interacción de semilla por estado.

\begin{tabular}{|c|c|c|c|c|c|c|}
\hline \multicolumn{7}{|c|}{ LSmean de las Semillas } \\
\hline Semilla & & Media \pm E.E**. & G.L. & Valor de $\mathrm{t}$ & $\operatorname{Pr}>|\mathrm{t}|$ & letras \\
\hline Arveja & & $37,5 \pm 1,97$ & 1548 & 19,06 & $<0,0001$ & C \\
\hline Maíz & & $51,3 \pm 1,97$ & 1548 & 26,09 & $<0,0001$ & B \\
\hline Trigo & & $63,1 \pm 1,97$ & 1548 & 32,06 & $<0,0001$ & A \\
\hline \multicolumn{7}{|c|}{ LSmean por Estado de la semilla } \\
\hline Estado & & Media \pm E.E. & G.L. & Valor de $\mathrm{t}$ & $\operatorname{Pr}>|\mathrm{t}|$ & letras \\
\hline Seca & & $53,3 \pm 1,61$ & 1548 & 33,2 & $<0,0001$ & A \\
\hline Hidratada & & $47,9 \pm 1,61$ & 1548 & 28,84 & $<0,0001$ & B \\
\hline \multicolumn{7}{|c|}{ LSmean de las Semillas $x$ Estado (secas e hidratadas) } \\
\hline Semilla & Estado & Media \pm E.E. & G.L. & Valor de $\mathrm{t}$ & $\operatorname{Pr}>|\mathrm{t}|$ & letras \\
\hline Arveja & Seca & $25,0 \pm 2,78$ & 1548 & 8,98 & $<0,0001$ & $\mathrm{D}$ \\
\hline Arveja & Hidratada & $50,0 \pm 2,78$ & 1548 & 19,97 & $<0,0001$ & B \\
\hline Maíz & Seca & $64,6 \pm 2,78$ & 1548 & 23,22 & $<0,0001$ & A \\
\hline Maíz & Hidratada & $38,1 \pm 2,78$ & 1548 & 13,68 & $<0,0001$ & C \\
\hline Trigo & Seca & $70,4 \pm 2,78$ & 1548 & 25,29 & $<0,0001$ & A \\
\hline
\end{tabular}

*LSmeans (mínimo de los cuadrados de la media) Proc Glimmx, P=0.05. Números seguidos por la misma letra no presentan diferencias significativas.

** E. E. Error Estándar

con valores de (20\%). La destrucción de las semillas se empezó a presentar a partir de las 18 horas (25\%) y aumentó hasta las 72 horas, con valores del $40 \%$.

En la evaluación de la germinación, luego de presentarse el daño, se observó que las semillas que sufrieron lesiones en el endospermo, alcanzaron a germinar; sin embargo, el daño puede inducir desórdenes sanitarios, por el ataque de hongos patógenos o saprofitos o la pérdida de vigor de la planta. Dependiendo de la intensidad de la lesión sobre el endospermo, el daño puede ser tolerable, si es pequeño y, en bajas proporciones, en el lote de semillas. La lesión se consideró grave, cuando se presentó sobre el embrión o el ataque causa la destrucción completa de la semilla, ya que en ambos casos se evidenció la pérdida total. En conjunto, considerando las partes de la semilla atacadas (embrión y destrucción de la semilla), alcanzaron valores de 38,2\% (Tabla 1), que se considera de importancia económica, por la pérdida de producción o por los costos de resiembra y valor de estas labores y remplazo de la semilla y demás insumos utilizados en la siembra.

En esta investigación. la evaluación demostró que las semillas más susceptibles al daño ocasionado por las larvas del falso gusano alambre fueron las de trigo, seguido por las semillas de maíz y, finalmente, las de arveja, como lo indica la frecuencia de las observaciones. Por estado de las semillas, secas e hidratadas, las más susceptibles fueron las de trigo y de maíz secas, seguidas por las de trigo y de maíz hidratadas y las menos susceptibles fueron las de arveja secas e hidratadas (Tabla 3).

Si bien el estado de la semilla de maíz y de trigo hidratadas puede contribuir a la rápida germinación de la semilla y a recibir una menor proporción del daño, igualmente, es posible que el estímulo de la rápida germinación contribuya a que reciba mayor ataque, ya que en el proceso de germinación las semillas liberan compuestos volátiles, que le permiten a 
Tabla 3. LSmeans* de la respuesta general del daño sobre las semillas por estado, secas e hidratadas, a diferentes tiempos de evaluación.

\begin{tabular}{|l|l|l|l|l|l|l|l|}
\hline Semilla & \multicolumn{4}{l}{ Arveja } & \multicolumn{2}{l|}{ Maíz } & \multicolumn{2}{l|}{ Trigo } \\
\hline Horas & $\mathbf{N}$ & $\begin{array}{l}\text { Seca } \\
\text { Media } \pm \text { E.E. }\end{array}$ & $\begin{array}{l}\text { Media } \pm \text { E.E } \\
\text { Media } \pm \text { E.E }\end{array}$ & $\begin{array}{l}\text { Medida } \pm \text { E.E } \\
\text { Media } \pm \text { E.E }\end{array}$ & Media \pm E.E \\
\hline 0 & 20 & $0 \pm 0,0 \mathrm{D}$ & $0 \pm 0,0 \mathrm{~B}$ & $0 \pm 0,0 \mathrm{E}$ & $0 \pm 0,0 \mathrm{~B}$ & $0 \pm 0,0 \mathrm{C}$ & $0 \pm 10,0 \mathrm{~B}$ \\
\hline 6 & 20 & $10 \pm 6,9 \mathrm{CD}$ & $40 \pm 11,2 \mathrm{~A}$ & $25 \pm 9,9 \mathrm{DE}$ & $35 \pm 10,9 \mathrm{~A}$ & $50 \pm 11,5 \mathrm{BC}$ & $55 \pm 11,4 \mathrm{~A}$ \\
\hline 12 & 20 & $10 \pm 6,9 \mathrm{CD}$ & $45 \pm 11,4 \mathrm{~A}$ & $50 \pm 11,5 \mathrm{CD}$ & $35 \pm 10,9 \mathrm{~A}$ & $70 \pm 10,5 \mathrm{BC}$ & $55 \pm 11,4 \mathrm{~A}$ \\
\hline 18 & 20 & $15 \pm 8,2 \mathrm{CD}$ & $45 \pm 11,4 \mathrm{~A}$ & $60 \pm 11,2 \mathrm{BC}$ & $35 \pm 10,9 \mathrm{~A}$ & $80 \pm 9,2 \mathrm{~A}$ & $55 \pm 11,4 \mathrm{~A}$ \\
\hline 24 & 20 & $20 \pm 9,2 \mathrm{BCD}$ & $55 \pm 11,4 \mathrm{~A}$ & $60 \pm 11,2 \mathrm{BC}$ & $35 \pm 10,9 \mathrm{~A}$ & $85 \pm 8,2 \mathrm{~A}$ & $55 \pm 11,4 \mathrm{~A}$ \\
\hline 30 & 20 & $20 \pm 9,2 \mathrm{BCD}$ & $55 \pm 11,4 \mathrm{~A}$ & $65 \pm 10,9 \mathrm{ABC}$ & $40 \pm 11,2 \mathrm{~A}$ & $85 \pm \mathrm{a}, 2 \mathrm{~A}$ & $55 \pm 11,4 \mathrm{~A}$ \\
\hline 36 & 20 & $20 \pm 9,2 \mathrm{BCD}$ & $55 \pm 11,4 \mathrm{~A}$ & $65 \pm 10,9 \mathrm{ABC}$ & $45 \pm 11,4 \mathrm{~A}$ & $90 \pm 6,9 \mathrm{~A}$ & $60 \pm 11,2 \mathrm{~A}$ \\
\hline 42 & 20 & $25 \pm 9,9 \mathrm{BCD}$ & $60 \pm 11,4 \mathrm{~A}$ & $80 \pm 9,2 \mathrm{AB}$ & $45 \pm 11,4 \mathrm{~A}$ & $90 \pm 6,9 \mathrm{~A}$ & $65 \pm 10,9 \mathrm{~A}$ \\
\hline 48 & 20 & $30 \pm 10,5 \mathrm{BC}$ & $60 \pm 11,2 \mathrm{~A}$ & $85 \pm 8,2 \mathrm{AB}$ & $45 \pm 11,4 \mathrm{~A}$ & $90 \pm 6,9 \mathrm{~A}$ & $65 \pm 10,9 \mathrm{~A}$ \\
\hline 54 & 20 & $35 \pm 10,9 \mathrm{ABC}$ & $60 \pm 11,2 \mathrm{~A}$ & $85 \pm 8,2 \mathrm{AB}$ & $45 \pm 11,4 \mathrm{~A}$ & $90 \pm 6,9 \mathrm{~A}$ & $65 \pm 10,9 \mathrm{~A}$ \\
\hline 60 & 20 & $35 \pm 10,9 \mathrm{ABC}$ & $60 \pm 11,2 \mathrm{~A}$ & $85 \pm 8,2 \mathrm{AB}$ & $45 \pm 11,4 \mathrm{~A}$ & $90 \pm 6,9 \mathrm{~A}$ & $65 \pm 10,9 \mathrm{~A}$ \\
\hline 66 & 20 & $45 \pm 11,4 \mathrm{AB}$ & $60 \pm 11,2 \mathrm{~A}$ & $90 \pm 8,9 \mathrm{~A}$ & $45 \pm 11,4 \mathrm{~A}$ & $90 \pm 6,9 \mathrm{~A}$ & $65 \pm 10,9 \mathrm{~A}$ \\
\hline 72 & 20 & $60 \pm 11,2 \mathrm{~A}$ & $60 \pm 11,2 \mathrm{~A}$ & $90 \pm 6,9 \mathrm{~A}$ & $45 \pm 11,4 \mathrm{~A}$ & $90 \pm 6,9 \mathrm{~A}$ & $65 \pm 10,9 \mathrm{~A}$ \\
\hline
\end{tabular}

*LSmeans (mínimo de los cuadrados de la media) Proc Glimmx, P=0,05. Números seguidos por la misma letra no presentan diferencias significativas.

los insectos ubicarlas, lo cual, se ha demostrado para insectos trozadores, como el gusano alambre (Doane et al. 1975; Doyen \& Tschinkel, 1974; Horton \& Landolt, 2002); sin embargo, sobre las semillas que alcanzaron a germinar y tenían algún daño, se desarrollaron hongos, como Fusarium sp. y Pencilcilium sp. (Figura 2), que pueden llegar a ser responsables de pérdida de vigor de las plántulas, por competencia por nutrientes, contribuir al desarrollo de micotoxinas o evitar el establecimiento de endófitos, que contribuyen a la protección de la planta (Bacon et al. 2001). Estos aspectos justifican dedicar mayores esfuerzos de investigación para alcanzar una visión completa del efecto del daño ocasionado por el falso gusano alambre sobre sobre las semillas-plántulas de arveja, de maíz y de trigo evaluadas.

Los resultados indican que las larvas del falso gusano alambre, se alimentan de las semillas, inmediatamente éstas se colocan el suelo y no les dan tiempo de hidratarse y de ger- minar, por lo que en suelos, con presencia de este insecto, las pérdidas de la población de plantas puede llegar a afectar severamente el desarrollo de los cultivos.

Para el manejo de este insecto, se debe considerar que el daño que sufren las semillas, por el ataque de las larvas, puede alcanzar importancia económica, por la pérdida total de la semilla o porque afecta el desarrollo de la planta. El daño, se encontró que afecta severamente las semillas de trigo y de maíz y, moderadamente, a las semillas de arveja.

Adicionalmente, se debe tener en cuenta la pérdida de vigor de las plantas por acción directa del insecto y las consecuencias, como stress hídrico o volcamiento por la pérdida del sistema radicular. A causa de esto, en los lotes, con historia de la presencia del falso gusano alambre, se puede tomar la decisión de ser sembrados con semilla de óptima calidad (certificada), tratada con un insecticida, emplear cebos en- 
venenados (tóxicos) (Zenner de Polanía et al. 1999) y realizar una adecuada preparación del suelo, complementada con medidas de labores culturales, como el aseo del lote (remoción de escombros, residuos vegetales de cosechas o de control de malezas), para prevenir que los adultos encuentren sitios donde refugiarse y reproducirse (Quiroga \& Posada, datos aún no publicados).

Agradecimientos. Los autores expresan sus agradecimientos a la Universidad de Ciencias Aplicadas y Ambientales U.D.C.A, por el apoyo brindado a esta investigación realizada dentro del semillero Entomi dedicati, adscrito al grupo de investigación de Fitosanidad. Conflicto de intereses: El manuscrito fue preparado y revisado con la participación de todos los autores, quienes declaramos que no existe ningún conflicto de intereses, que ponga en riesgo la validez de los resultados presentados.

\section{BIBLIOGRAFÍA}

1. BACON, C.W.; YATES, I.E.; HINTON, D.M.; MEREDITH, F. 2001. Biological control of Fusarium moniliforme in maize. Environm. Health Perspect. 109(2):325332.

2. DOANE, J.F.; LEE, Y.W.; KLINGLER, J.; WESTCOTT, N.D. 1975. The orientation response of Ctemcera destructor and other wire worms (Coleoptera: Elateridae) to germinating grain and to carbon dioxide. Can. Entomol. 107(12):1233-1252.

3. DOYEN, J.T.; TSCHINKEL, W.F. 1974. Population size, microgeographic distribution and habitat separation in some tenebrionid beetles (Coleoptera). Ann. Entomol. Soc. Am. 67:617-626.

4. ESPINAL T., L.S.; MONTENEGRO M., E. 1977. Zonas de vida o formaciones vegetales de Colombia. Memoria explicativa sobre el mapa ecológico de Colombia. Instituto Geográfico Agustín Codazzi, IGAC. Departamento Agrológico, Bogotá. 238p.

5. HORTON, D.R.; LANDOLT, P.J. 2002. Orientation response of Pacific coast wireworm (Coleoptera: Elateridae) to food baits in laboratory and effectiveness of baits in field. Can. Entomol. 134(3):357-367.

6. LÓPEZ A., A.; ESPITIA M., E.; RONDÓN C., G.; ARRIETA H., J.M. 1995. La de todos los años. NOTAS Y NOTICIAS ENTOMOLOGICAS Boletín del Programa Nacional de Manejo Integrado de Plagas. CORPOICA. 1(2) 13.
7. McINTYRE, N.E. 1998. Pitfall Trapping of Male Darkling Beetles Not Induced by Females. The Prairie Naturalist 30(2): June 1998. Disponible desde internet en: http://www.biol.ttu.edu/faculty/nmcintyre/research/ prairienat30_2_1998.pdf (con acceso 23/12/12).

8. METCALF, C.L.; FLINT, W.P. 1962. Destructive and useful insects: their habit and control. New York: London: Mc Graw Hill, 1087p.

9. POSADA, L.; ZENNER DE POLANÍA, I.; CALVACHE G., H.; BORRERO F., F. 1988. Impacto visual. Notas y Noticias Entomológicas (NNE). Programa de Entomología. I.C.A. Enero - Febrero 1988:4.

10. POSADA, L.; ZENNER DE POLANÍA, I.; CALVACHE G., H.; RODRÍGUEZ S., D.A. 1987. Falso gusano alambre. Notas y Noticias Entomológicas (NNE). Programa de Entomología. I.C.A. Mayo - Junio 1987:3.

11. POSADA O, L.; ZENNER DE POLANÍA, I.; LÓPEZ, A.; RUIZ, N. 1980. Al fin se conoce huésped. Notas y Noticias Entomológicas (NNE). Programa de Entomología. I.C.A. Julio - Agosto 1980:2.

12. POSADA O., L.; ZENNER DE POLANÍA, I.; BORRERO F., F. 1990a. Ya se acerca. Notas y Noticias Entomológicas (NNE). Programa de Entomología. I.C.A. Septiembre - Octubre 1990:1.

13. POSADA O, L.; ZENNER DE POLANÍA, I.; BORRERO F., F. 1990b. Si son plagas. Notas y Noticias Entomológicas (NNE). Programa de Entomología. I.C.A. Mayo - Junio 1990:1.

14. POSADA O., L. 1989. Lista de insectos dañinos y otras plagas en Colombia. Bogotá: Instituto Colombiano Agropecuario, Bol. Técn. No. 43. 662p.

15. SAS INSTITUTE. 2009. SAS user's guide: statistics, version. 9.1.3. SAS Institute, Cary, NC.

16. TOBA, H.H. 1985. Damage to potato tubers by false wireworm larvae. J. Agric. Entomol. 2(1):13-19. Disponible desde internet en: http://scentsoc.org/ Volumes/JAE/v2/1/00021013.pdf (con acceso 23/12/12).

17. VERGARA, R.; VARELA, A.L. 1977. Plaga potencial? Notas y Noticias Entomológicas (NNE). Programa de Entomología. I.C.A. Septiembre - Octubre. 1977:18. 
18. ZENNER DE POLANÍA, I. 1994a. Por punta y punta. Notas y Noticias Entomológicas (NNE). Programa de Entomología. I.C.A. Enero-Junio 1994:1.

19. ZENNER DE POLANÍA, I. 1994b. También le hala. Notas y Noticias Entomológicas (NNE). Programa de Entomología. I.C.A. Enero-Junio 1994:2.

20. ZENNER DE POLANÍA, I.; BORRERO F., F. 1992. Hábito adicional. Notas y Noticias Entomológicas (NNE).
Programa de Entomología. I.C.A. Marzo-abril. 1992:19.

21. ZENNER DE POLANÍA, I.; ÁLVAREZ R., A.; DUARTE G., W. 1999. No solo contra trozadores. Notas y Noticias Entomológicas (NNE). SOCOLEN. 27(3):4.

Recibido: Julio 19 de 2013

Aceptado: Octubre 21 de 2013

Como citar:

Quiroga-Murcia, D.; Posada-Flórez, F.J. 2013. Daño ocasionado por el falso gusano alambre Eleodes pos. omissoides Blaisdell (Coleoptera: Tenebrionidae) en semillas de gramíneas y leguminosas. Rev. U.D.C.A Act. \& Div. Cient. 16(2): $391-400$. 